\title{
Narrar, informar, conquistar: Los Viajes de Juan de Mandevilla en Aragón.
}

\section{Rodríguez Temperley, María Mercedes.}

Cita:

Rodríguez Temperley, María Mercedes (2001). Narrar, informar, conquistar: Los Viajes de Juan de Mandevilla en Aragón. Studia Neophilologica, 73, 184-196.

Dirección estable: https://www.aacademica.org/maria.mercedes.rodriguez.temperley/39 ARK: https://n2t.net/ark:/13683/pHWM/grc

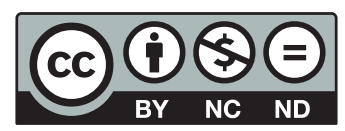




\title{
Narrar, Informar, Conquistar: Los Viajes de Juan de Mandevilla en Aragon
}

\author{
MARÍA MERCEDES RODRÍGUEZ TEMPERLEY
}

El Libro de las maravillas del mundo de Juan de Mandevilla, libro de viajes escrito originariamente en Francia, ingresa a la península a través de la Corona de Aragón. En efecto, la copia más antigua conservada en España es un manuscrito aragonés de fines del siglo XIV. En líneas generales, en lo referente a la producción del texto, se ha hecho demasiado hincapié en el carácter ficticio del viaje (y por lo tanto la composición meramente libresca del mismo); en cuanto a su recepción, se ha privilegiado su carácter maravilloso (en tanto repertorio de mirabilia) fundamentalmente destinado a una lectura de pasatiempo, y hasta se lo ha relacionado con cierto gusto por las "extravagancias" manifestado por Juan I de Aragón.

Tales interpretaciones son el resultado de lecturas aisladas que desdibujan o empañan otras posibles funcionalidades de dicho texto en el reino de Aragón a fines del siglo XIV. El presente trabajo busca dar cuenta de datos históricos que refuerzan la idea de una lectura de tipo informativa tendiente a recabar datos acerca de Tierra Santa y otras regiones de Oriente, en el marco de los intereses expansionistas de la corona de Aragón.

\section{NARRAR. Un libro de maravillas: lectores medievales y modernos}

Del conjunto de libros de viajes conservados en la España medieval, existe uno en particular que ha pasado a la historia como la obra de un "gran farsante", de un viajero de cámara que sólo viajó a través de los libros de sus predecesores. Hablamos de Sir John Mandeville o Juan de Mandevilla, con cuyo nombre se lo conoció en la península. Su Libro de las Maravillas del Mundo, escrito originariamente en francés alrededor de 1360, incluye un itinerario a Tierra Santa y un recorrido por regiones de Oriente, China, norte de Africa y las tierras del Preste Juan, realizado supuestamente por el autor a mediados del siglo XIV.

Aunque tuvo una enorme difusión en toda Europa ininterrumpidamente durante casi tres siglos según demuestran los casi trescientos manuscritos conservados en diversas lenguas europeas, en España se conocen sólo una versión aragonesa de fines del siglo XIV (de la que nos ocuparemos en nuestro trabajo) ${ }^{1}$ y varias ediciones castellanas del siglo $\mathrm{XVI}^{2}$. Se conserva también un fragmento en catalán, inserto dentro de otra obra, el Thrésor de l'Histoire des langues de cest. Univers o Histoire admirable des plantes et herbes de Claude Duret ${ }^{3}$.

La bibliografía sobre Mandevilla no deja de ser vasta; una puntualización casi constante es la referida a su carácter de viaje no-real. Por ejemplo, se habla de la "inexistencia de un fondo original" del relato producto de la refundición y acopio de otros relatos con los que configura su viaje (Richard, 1985: 90), de "descripciones falsas" de Armenia, Mongolia y China (Fick, 1976: 23), de "superchería" (Manuel Gómez Moreno y Mata Carriazo, 1962: xxix), de "suma de noticias tomadas de diferentes fuentes (calladas siempre) y enhebradas a través de un viaje que el autor cuenta haber realizado" (López Estrada, 1999: 14). Afortunadamente, la crítica ha ido advirtiendo cada vez más sobre la inconveniencia de tales juicios, reformulando sus postulados en una orientación cercana a lo que Lee Patterson (1987) denomina "negociar el pasado". Todo intento de reconstrucción del pasado conlleva una constante "negociación", ya que tanto la necesidad de captar las diferencias de una cultura o de un momento histórico, como la propia cultura o momento 
histórico del sujeto que interpreta, se encuentran en juego simultáneamente cuando se acercan al objeto de estudio. Dado que la orientación del presente trabajo se dirige a evaluar la posibilidad de una lectura en clave política del texto mandevillesco, se intentará dar muestras de ello a través de la organización de datos históricos que, puestos en una visión de conjunto, permitirían fundamentar dicha funcionalidad en el reino aragonés a fines del siglo XIV.

Tanto Waters Bennett (1954), cuyo estudio sobre el libro de Mandevilla incluye apéndices con las descripciones de los manuscritos existentes, como Liria Montañés (1979), editora de la versión aragonesa ${ }^{4}$, se limitan a señalar, siguiendo a Entwistle (1922), que los testimonios más tempranos referidos a la entrada de Mandevilla en España son los pedidos de Juan I de Aragón al rey de Francia el 13 de agosto de 1380 y a la duquesa de Bar el 20 de octubre del mismo año. Ninguna de las estudiosas va más allá acerca de las causas que pudieron haber motivado dichas solicitudes, excepto la necesidad de contar con una traducción a la lengua vernácula en la que el texto francés habría servido como modelo.

William Entwistle (1922: 251), en un trabajo temprano e ineludible, se refiere al patronazgo literario que Juan I de Aragón había heredado de su padre, Pedro el Ceremonioso, y al interés personal, lindante en lo morboso ("verging on the morbid"), por los viajes lejanos, por el conocimiento de lo oculto y por la información sobre las cosas extrañas del mundo. Al respecto, exhibe ejemplos de tal interés real manifestado en títulos de su biblioteca obtenidos en distintos años, como De locis Terrae Sanctae de Theodorico (1374), De mirabilibus Terrae Sanctae de Odorico (1378) y por último, Lo Purgatori de San Patrici de Mandrevila (1395), según figura en el inventario de la biblioteca real aunque se trata, en realidad, de dos obras distintas ${ }^{5}$.

Alda Rossebastiano (1997) comparte algunos criterios de su colega inglés, y en su estudio sobre la tradición ibero-románica del Libro de las maravillas del mundo, se refiere al interés originado en el ambiente cortesano đe la península ibérica, unido al gusto por la extravagancia y a la curiosidad por la magia y la superstición que debieron guiar a Juan I para solicitar las copias del texto francés (p. 8). Parecería que las hipótesis de Entwistle y Rossebastiano buscan evidencias que refuercen una "lectura de lo maravilloso" como una forma de explicar el interés suscitado por el texto en la casa de Aragón. Al respecto, no descartamos dichas interpretaciones pero las consideramos insuficientes, ya que soslayan otras posibles funcionalidades que el texto pudo haber tenido en el reino de Aragón a finales del siglo XIV. Más allá del interés librado a caprichos reales, a una morbosa curiosidad o a la voluntad coleccionista de un monarca, los trabajos críticos hasta ahora expuestos se limitan a enunciar sólo las circunstancias y los personajes involucrados en el proceso de adquisición del texto, sin preguntarse en ningún momento por motivaciones más profundas (de tipo histórico, ideológico o político) que pudieron haber guiado las circunstancias de su petición por partida doble, tal como hemos puntualizado.

\section{INFORMAR. Relatos, mapas, astrolabios}

La comprensión de los textos medievales requiere la reconstrucción del contexto en que fueron escritos y leídos. Dicha reconstrucción, no siempre posible, permite vislumbrar una organización distinta a la construida por nuestros preconceptos modernos. Por ello, parece oportuno rescatar el concepto de Paul Zumthor (1972: 19-31) acerca de la "doble historicidad" de los textos medievales, orientada al mismo tiempo hacia los procesos de producción y recepción de estos productos culturales. En tal sentido, la historia y sus documentos nos permiten avanzar en la restauración original del significado del texto con el objeto de recuperar la lectura del texto mandevillesco en la corte aragonesa durante aquellos años. En este trabajo intentaremos demostrar la importancia de este libro en la corte aragonesa, la cual, envuelta en un marco de mesianismo político, realizaba intentos de expansión hacia Oriente y de recuperación de Tierra Santa. 
Con este objetivo, hemos de llamar la atención sobre tres cuestiones vinculadas entre sí durante los reinados de Pedro IV y Juan I de Aragón: 1) la obtención de otros libros o noticias con contenido similares acerca de Tierra Santa y regiones de Oriente, 2) el interés manifestado por instrumentos científicos de uso geográfico y astronómico y, por último, 3) el desarrollo de la cartografía, con el fin de establecer hasta qué punto estos aspectos constituyeron parte de un programa político destinado a obtener información sobre el lejano Oriente con vistas a reforzar la presencia aragonesa en Tierra Santa y sus alrededores. Interesa además evaluar la posibilidad de contacto con pueblos asiáticos, en particular los mongoles, cuya finalidad última consistía en establecer una alianza para enfrentar al poder turco, todo ello en el marco de una política expansionista que se verá retrasada con la pérdida de Morea y finalmente, por el avance conquistador de Castilla a través de sus expediciones atlánticas.

En lo referido al primer punto, ya hemos señalado los cuatro títulos indicados por Entwistle (De locis Terrae Sanctae de Theodorico, De mirabilibus Terrae Sanctae de Odorico, Lo Purgatori de San Patrici y el Libro de Mandevilla). A ellos habría que agregar, en primer término, el Libro de Marco Polo y la Flor de las ystorias de Orient, de Aytón de Gorigos. En tal sentido, exponemos a continuación una brevísima síntesis de la materia y circunstancias de realización de las mencionadas obras, con el objeto de mostrar los vínculos temáticos que las unen y obtener, de ese modo, cabal idea de hasta qué punto este grupo de textos formó parte de un programa literario-informativo-político sobre cuestiones de Oriente.

La obra de Theodorico, De locis Terrae Sanctae, (1169-1171) es, según Jean Richard (1985: 17, 46), la más destacada dentro de las de su grupo. En ella, el autor trata de completar el itinerario que otro peregrino había realizado a través de Judea, Galilea y Fenicia, y recomienda al peregrino seguir en su itinerario las etapas de la vida de Cristo, Betania, el huerto de Getsemaní, el camino del Calvario y el monte de los Olivos, luego de lo cual propone varios itinerarios para ir a Jerusalén y uno para llegar hasta Fenicia.

Tras doce años de travesía (1318-1330), el fraile franciscano Odorico de Pordenone regresa a Italia y dicta su relación de viaje, De mirabilibus Terrae Sanctae, a Guglielmo de Solagna, hermano de su orden. Su relato registra aspectos de su viaje a China y Tartaria (en especial sus vivencias y observaciones en la corte del Gran Khan) y es una de las fuentes reconocibles en el relato de Juan de Mandevilla.

Una novedad temática y espacial presenta Lo Purgatori de San Patrici. El purgatorio de San Patricio era una cueva situada en una isla del lago Dorg, en el norte de Irlanda, donde se creía que era posible acceder a una visión del Purgatorio. Cuando Juan I de Aragón murió súbitamente durante una cacería en 1396, Ramón de Perellós, militar y diplomático al servicio de Francia y Aragón, emprendió un viaje en septiembre de 1398 a Irlanda "para entrevistar al alma del monarca, saber si estaba en vía de salvación y aclarar así la cuestión de su muerte" (Taylor, 1991: 64). De su viaje y posterior entrevista con el alma del monarca, da testimonio su Viatge al Purgatori de Sant Patrici, traducción catalana del Tractatus Sancti Patricii de Hugo de Saltrey (c. 1185-1190), a la que le agrega el relato de su viaje y el encuentro con el alma del monarca.

Del Libro de Marco Polo, quizás el relato de viaje más emblemático, se registran los pagos de un ejemplar en octubre de 1372 y de dos ejemplares en febrero de 1374 y el envío como regalo del infante Juan al conde de Foix en 1384 (Rubió y Lluch, 1921: docs. CLXXIII, CLXXX). Existen traducciones al catalán y aragonés, esta última llevada a cabo por Juan Fernández de Heredia ${ }^{6}$ y conservada en el mismo códice que guarda el texto de Aytón.

La Flor de las ystorias de Orient, obra de clara intención propagandística, combinaba datos geográficos e históricos sobre Oriente, referencias sobre los tártaros y un tratado con un plan de cruzada para la recuperación de los Santos Lugares. Según Hauf i Valls (1996: 130), editor de la versión catalana, "la tesis sostenida por Aytón era que Occidente se había desentendido por completo de Tierra Santa al no saber aprovechar la oportunidad, tantas 
veces repetida, de jugar la carta de una ventajosa colaboración con los tártaros”. Escrita originariamente en francés en 1307, es traducida con anterioridad a 1396 también por Fernández de Heredia al catalán y al aragonés aduciendo razones políticas y religiosas: "Don fray iohan ferrandez de redia / maestro dela orden de sant iohan de / Gerusalem conssiderando quelas cosas contenidas es esti / present volumen podrian conel fauor de dios Redmidiar / en muyt grant prouecho et sobirana victoria del xpistianidat / et ensalçamiento de la nuestra fe catholica mando screuir / aquesti present libro...."7.

Si tomamos en cuenta el carácter pseudo-enciclopédico que guardaban estos textos en la Edad Media, podríamos agregar la traducción al catalán del Libro del Tesoro de Brunetto Latini realizada por Guillem de Copons a pedido de Pere d'Artés, consejero y "mestre racional" para Juan I de Aragón y su mujer, Violeta de Bar $^{8}$, y la petición del infante Juan al prior de los Predicadores de un ejemplar del compendio de Vicente de Beauvais en octubre de 1385 para su posterior traducción al catalán (Rubió y Lluch, 1921: CCXIV). Estos compendios enciclopédicos constituían uno de los abrevaderos de datos geográficos de obligada consulta para quienes deseaban recabar noticias sobre tierras lejanas, sus habitantes, creencias y costumbres e interactuaban con relatos de viajeros. Tal es el caso de Vicente de Beauvais, quien inserta en su Speculum Historiale la Historia Mongalorum, aquella parte del relato de viaje de fray Juan de Plano Carpino (1258), enviado del Papa a los tártaros, referida a la vida, costumbres y religión del pueblo mongol.

Curiosamente, la posesión de algunos de estos títulos en bibliotecas de otras cortes europeas, nos advierten acerca de intenciones políticas semejantes a las que tratamos de ilustrar. Al respecto, Patrick Winter (1985: 52), estudioso de la biblioteca de Philippe Le Hardi (1364-1404), duque de Borgoña y hermano del rey Carlos V de Francia, afirma lo siguiente:

\footnotetext{
'L'on trouve, dans la collection ducale, neuf manuscrits de récits de croisades, sujet appropié pour un prince qui devait être l'instigateur d'une expédition de ce genre. Parmi ces volumes se trouvent les chroniques de Guillaume de Tyr relatant les prouesses de Godefroi de Bouillon en Terre Sainte, dans un précieux manuscrit transcrit à Saint-Jean-d'Acre à la fin du XIIIe. siècle. Les exemplaires des chroniques de Villehardouin et d'Henri de Valenciennes provenaient de la famille de la duchesse. On connaît le succès presque immédiat de ces oeuvres, qui furent peut-être récitées, telles des chansons de geste, dans les châteaux. Au nombre des récentes acquisitions ducales figuraient la Fleur de les Histoires d'Hayton, le Voyage d'Outremer de Jean de Mandeville, ainsi que le Livre des Armoilles, tous ouvrages qui ont une relation avec les croisades. Dans l'inventaire de la duchesse, on relève aussi le Livre de Guillaume de Machaut. Il est probable qu'on pouvait lire, à la fin du manuscrit, la biographie de Pierre Ier. de Lusignan, roi de Chypre, qui avait pris Alexandrie en $1365^{\prime \prime}$
}

Al interés bibliográfico se le suma el aporte brindado por historias orales de viajeros o habitantes de tierras orientales cuyos testimonios constituían un corpus de noticias actualizadas. En tal sentido, el infante Juan ordena la traducción del relato que una mujer hiciera en Chipre a Bernat d'Anglesola, recién llegado de Tierra Santa, acerca de las maravillas existentes en su tierra, India: "porque volem que haiats traslat de tot ço que dit 1 en ha e que l nos aportets" (Rubió y Lluch, 1921: CCXXI) ${ }^{9}$. El 11 de septiembre de 1379 le encargaba al procurador real de Mallorca que por escrito le explicase todo lo que contaba un mallorquí que había arribado de Tartaria (Rubió y Lluch, 1908: CCCIII); en abril de 1391, ya rey, requería al conde de Foix que le enviara un fraile menor que contaba maravillas de la tierra del Preste Juan (Rubió y Lluch, 1908: CCCCXI) y años más tarde, en abril de 1394, solicitaba la presencia de un hombre proveniente de las tierras del gran Khan de Tartaria con el objeto de obtener noticias de aquellas partes del mundo (Rubió y Lluch, 1947: DCXLVII).

Hemos aludido también al interés suscitado por instrumentos científicos relacionados con la actividad geo-cartográfica. Innumerables son los documentos en los que Pedro IV y Juan I encargan astrolabios, relojes, cuadrantes y esferas (Rubió y Lluch, 1908, docs. CCII, CCLXV, CCLXXXIV, CCCXII, CCCCXXXI y 1921: docs. LXXVIII, CXXIX, 
CXLI, CXLIII, CXLIX, CLXXII, CCXVII, CCXXXV, CCXXXVI, CCXXXVII, CCXXXIX, CCLIII, CCLVIII, CCLXVIII, CCXC, CCCII, CCCXXXV), así como también libros sobre astrología o manuales de uso de los citados instrumentos (Rubió y Lluch, 1908: docs. CCIII y CCCIV, y 1921: docs. CLXXX, CLXXXVII, CCXXIV, CCXLII, CCXLV) ${ }^{10}$. Tampoco es casual la compilación de las Tablas de Barcelona (a semejanza de las Tablas Alfonsíes), todo ello enmarcado en la tradición que los estudios cosmográficos y astronómicos habían tenido a lo largo de la Edad Media en la confederación catalano-aragonesa. Como señala Millás Vallicrosa (1960: 299), "sus contactos con la cultura árabe y judaica, la invitación que la amplitud de sus costas daba para los viajes marítimos, todo ello explica el particular florecimiento de tales estudios hasta los mismos días renacentistas del reinado de Fernando el Católico". Como veremos más adelante, este interés, unido a lo que expondremos a continuación, delimitan un contexto favorable para la recepción de noticias atinentes a tierras lejanas o a otras partes del mundo.

Por último, nos referiremos al desarrollo de la cartografía mallorquina llevado a cabo bajo el reinado de estos dos monarcas a partir, sobre todo, de la obra de Abraham y Jafuda Cresques $^{11}$. Obra del primero de ellos es la famosa carta o portulano del año 1375 , considerado el mapamundi más moderno en su época, del cual quien luego sería el rey Carlos V de Francia solicita una copia a Pedro IV de Aragón. En dicho mapa se incluían todas las partes del mundo, para lo cual el cartógrafo debió acudir a la bibliografía de su tiempo: las narraciones de viajeros a Oriente de los siglos XIII y XIV. La importancia del atlas catalán es que por primera vez, Asia asume una forma reconocible ya que, si bien subsisten inexactitudes, difiere notablemente de aquellos mapas TO tan difundidos durante toda la Edad Media. Dos datos importantes a tener en cuenta son la afirmación casi segura de la esfericidad de la Tierra ${ }^{12}$, tópico que es tratado ampliamente en el libro de Juan de Mandevilla ("Car nuestro seynnor fezo la tierra Ronda enel medio logar del firmament", p. 134) y las numerosas leyendas que acompañan el trazado del mapa, que revelan la utilización de los relatos de Marco Polo, de Odorico y otros viajeros para la confección del mismo. Se conserva una nutrida documentación referida al tema: el 26 de abril de 1379 el infante Juan encarga un mapamundi a Mallorca (Rubió y Lluch, 1921:CCXII); el 5 de noviembre de 1381 Pere Palau entrega al enviado del rey de Francia, en nombre del infante Juan, un mapamundi realizado por Cresques (Rubió y Lluch, 1908: CCCXXII); en marzo de 1382 Pedro III ordena el pago a Abraham Cresques por "unes taules ont es representada la figura del mon" (Rubió y Lluch, 1921: CCLX), en 1387 se alude a un mapamundi comenzado por Abraham Cresques y que, a la muerte de éste, concluirá su hijo Jafuda (Rubió y Lluch, 1908: CCCLXXXVI) y en enero de 1391 Juan I pide un mapamundi y unas tablas de navegar (Rubió y Lluch, 1908: CCCCIX.

\section{CONQUISTAR. Intereses expansionistas}

Hasta aquí, algunos datos históricos que ayudan a contextualizar el posible interés suscitado por el texto de Juan de Mandevilla en cuanto a sus noticias sobre Tierra Santa y Oriente, y a su teoría sobre la redondez de la tierra comprobada a través del uso del astrolabio:

\footnotetext{
"Por que hombre puede bien apercebir que la tierra e la mar son de Redonda forma. Car la partida del firmamiento paresce en vna tierra que no paresce point en otra. Et esto puede hombre trobar por experiença e por sotil indagation, que si hombre trueba passage de nau e de gentes qui vayan ayr veer el mundo e de juso e de suso hombre podria yr nauegando todo entor el mundo de juso et de suso la quoal cosa yo prueuo assi segunt lo que yo he ensayado. Car yo he seido vers las partidas de braban. Et he Regoardado l'astralabio que la trasmontanna es .liij. grados d'alto. Et en alamaynna vers bohemia eilla .lviij. Et mas auant vers las partidas de Septentrion eilla ha .lxij. grados d'alto e algunos minutos. Car yo mesmo lo he mensurado al estrelabio" (p. 95).
} 
Sin embargo, al inicio de este trabajo aludimos a intereses expansionistas de Aragón hacia Oriente y la recuperación de los Santos Lugares. Las ideas de cruzada no eran nuevas en Aragón. Jaime I había intentado convencer, sin éxito, a Alfonso el Sabio para encarar una operación conjunta cuyo proyecto era el rescate de los Santos Lugares por medio de una alianza con los mongoles. A pesar de sus dos expediciones en 1269 y 1276, su idea de cruzada no arraigó pero sentó las bases de un clima de interés y movilización cultural cuyas especulaciones intelectuales más conocidas son las de Ramón Llull. En su Liber Tartari et Christiani (1282-1287) postula la conversión de los tártaros al cristianismo, para lo cual era necesario el aprendizaje de lenguas orientales por parte de los misioneros cristianos, idea que cuajará en el Concilio de Viena (1311-1312) cuando se ordene la creación de cátedras de lenguas orientales en las Universidades de París, Bolonia, Oxford, Salamanca y la Curia; años más tarde, en su Liber de Fine (1305), expondrá su proyecto para la recuperación de Tierra Santa y analizará las ventajas y desventajas de los posibles itinerarios. El proyecto de colaboración cristiano-mongólica existió en la corona de Aragón en tiempos de Jaime II (1291-1327), del mismo modo en que se avanza en la expansión catalano-aragonesa como lo atestiguan la famosa expedición de los catalanes a Oriente al mando de Roger de Flor en 1303, la fundación de los ducados de Atenas y Neopatria (1311-1312), y la incorporación de la isla de Cerdeña en 1324 como un reino más a la corona de Aragón. Con Pedro IV se lleva a cabo la reintegración del reino de Mallorca, luego de la derrota y muerte de Jaime III en 1349; paralelamente se desarrollan acciones de tipo diplomático, como las relaciones estrechas con Egipto, que llevaron a la obtención del protectorado sobre los reinos cristianos de Oriente y en cierta medida sobre los Santos Lugares ${ }^{13}$, lo cual facilitaba el acceso de los peregrinos a los mismos. Juan I (1387-1395) y Martín el Humano (1395-1410), sucesores de Pedro el Ceremonioso, aportarán subsidios económicos para la conservación de los santuarios de Tierra Santa, como signo de su interés por mantener la política de su predecesor ${ }^{14}$.

Llegado este punto se hace obligado mencionar, circunscribiéndonos al tema que nos ocupa, a un lucido personaje de aquel tiempo. Me refiero a Juan Fernández de Heredia (?1396), gran maestre de la Orden de los Hospitalarios, canciller y consejero de Pedro el Ceremonioso y amigo de Juan I de Aragón, sobre quien influyó marcadamente en el aspecto cultural y a quien ya nos hemos referido al mencionar sus traducciones de Marco Polo y de Aytón. Interesan sus acciones relacionadas con Oriente, entre las que se cuentan su ayuda a los venecianos en el rescate de la ciudad de Patrás, en manos de los turcos, que concluirá con la toma de Lepanto en 1378; la protección con los caballeros de su Orden de los ducados catalanes de Oriente; su expedición a Morea, iniciada con el propósito de detener el avance turco que, ejerciendo presión sobre Macedonia, dominaba casi toda Asia Menor, y que tendrá un final adverso del que no se salvará ni el mismo Heredia, al ser tomado como prisionero entre 1378 y 1379. Estudiosos como Luisa Ledesma Rubio, Juan Manuel Cacho Blecua, Albert Hauf i Valls y Miguel Cortés Arrese han coincidido en considerarlo como uno de los últimos cruzados de la etapa medieval, ya que como Gran Maestre de la Orden de San Juan de Jerusalén -cuya última finalidad era la recuperación de Tierra Santa-, como responsable de la estrategia papal, y como Gran Maestre de Rodas, la isla bastión de la cristiandad ante el avance turco, resulta difícil pensar que se resistiese al desvanecimiento de la utopía de conquistar Jerusalén.

Para quienes la idea de cruzada pueda sonar anacrónica en la segunda mitad del siglo XIV, debemos recordar que, como afirma Hauf i Valls (1996: 138), inspirándose en Atiya, "si sustituimos Egipto por el Imperio Otomano, y la Tierra Santa por Constantinopla, la idea continuará cautivando la imaginación de Occidente cristiano. Sin ir más lejos, el trágico hado de Armenia, representado por la figura de León VI de Armenia, hecho prisionero en 1375 y refugiado en Roma y París, era bien conocida a finales del siglo XIV. Y cuando, entre 1381 y 1405 , los mongoles de Tamerlán detuvieron temporalmente la invasión turca ante Constantinopla, la historia parecía repetirse cíclicamente". Al respecto, el mismo Aziz S. Atiya (1938: 12) se refiere a la cruzada a fines de la Edad Media como 
una preocupación viva para Occidente: "Even if Europe was not in the mood to make any further attempts to subdue the East and save the Holy Land from the unbeliever, it seemed as though a crusade would be needed to save Eastern and Central Europe from the Turkish menace".

En este contexto, propongo algunas calas del libro de Juan de Mandevilla, que reforzarían la información existente acerca de los pueblos mongoles y las posibilidades de una conversión derivables hacia una futura alianza. Las mismas deberían leerse en paralelo con las aseveraciones en sentido semejante expuestas por Aytón, Odorico, Marco Polo y Vicente de Beauvais.

- Predestinación y poderío:

"Et [Canguis] jazia vna noche en su lecho e dormia e vio vna vision qu'el venia deuant el .j. cauallero todo blanco armado d'armaduras blanquas caualgando sobre vn cauallo blanco, el li diso: 'can ¿duermes tu? A tu m'embia el dios jnmortal et es su voluntat que tu digas alos .vij. lignages que tu seas lur emperador. Car tu conqueriras las tierras que son aqui entor e seran comarcantes en tu subgeccion asi que tu has seido enla lur. Car esta es la voluntat de dios jnmortal". (p. 109)

- Origen cristiano de algunos Khanes:

"Et pues mango can qui fue buen christiano baptizado et dio letras de perpetual paz alos christianos. Et jmbia su hermano halaon con grant multitud de gentes por ganar la tierra santa et por la meter enlas manos delos christianos et por destruir la ley de mahomet e por prender el califfa de baudas qui hera seynnor e emperador de todos los moros. Et pues ouo halaon ganado toda la tierra de promission e puesta en manos delos christianos. Mas el gran Can en este comedio si fue delos aferes todo empaschado. Apres mango Can Regna cobila Can qui fue assi christiano e Regno .xlij. aynnos, el fundo la grant cibdat de jomg en cathay qui es mas grant assaz que no es Roma: l'otro grant Can que veno empues deuino pagano e todos los otros empues" (p. 111)

- Reverencia hacia la cruz de los cristianos:

"Et alla do los Religiosos christianos fincan assi como eillos facen en muchas cibdades en su tierra, eillos li van al deuant en procession con la cruz e l'agoa bendita, et cantan adalta voz: 'veni creator spiritus etc.'. Et quando eill los oye comanda alos seynnores que caualguen cerca d'eill que eillos fagan venir aquellos Religiosos. Et quando eillos s'acercan, e el vee la cruz, el tira su galeot que es sobre su cabeça (...) e de pues s'enclina ala cruz ...”. (p. 116)

- Tolerancia religiosa y poderío:

"Por desuso ni por de juso el firmament no ha point assi grant ni assi poderoso seynnor que el gran Can, ni de jus la tierra ni desuso. Car preste johan que es emperador del'alta jndia ni el soldan de Babilonia ni l'emperador de persia no han comparation aeill ni a su poder ni de noblezas ni de Riqueza. Car en todo esto eill passa todos los principes terrianos, de que es grant domage que el no cree en dios firmement. Et maguera eill oye muy volenters fablar de dios et sufre bien que sean christianos qui quiere por toda su tierra." (p. 116)

Habrá quienes intenten desestimar el texto de Mandevilla, argumentando que en su compilación de fuentes previas no aporta novedades respecto de Aytón, de Odorico o de Vicente de Beauvais, quien en su Speculum Historiale dedica varios capítulos a los mongoles (tomados, a su vez de Juan de Plano Carpino) con los ya conocidos tópicos referidos a sus costumbres, tolerancia en materia religiosa, modos de hacer la guerra y poderío. Dicho juicio implicaría desconocer el funcionamiento de la cultura medieval que, al compartir una misma lengua - el latín -, la Biblia como texto fundamental, origen y fin de todos los demás, y la traducción patrística como único testimonio de la cultura clásica, pareciera no decir nada nuevo en su comentario de comentarios y en su apelación constante a las autoridades. Por el contrario, como afirma Umberto Eco (1997: 11), "la cultura medieval tiene el sentido de la innovación, pero se las ingenia para esconderlo bajo el disfraz de la repetición (al contrario de la cultura moderna, que finge innovar incluso 
cuando repite)". En esta acumulación de testimonios, se apela a lo conocido y se busca una confirmación de lo ya dicho.

Pero los proyectos de conversión o alianza con los pueblos mongoles a los que hemos aludido no eran más que un recurso, una herramienta o un medio para alcanzar un objetivo último: la recuperación de los Santos Lugares y la lucha contra los enemigos cristianos. Aquí se produce un sugerente enlace con otro tema de gran raigambre en Aragón, y que me atrevería a colocar en estrecha relación con el ya mencionado de las Cruzadas: el. mesianismo político que, desde Arnaldo de Vilanova en adelante, calará hondamente en la época de los Reyes Católicos.

Como ha estudiado José Guadalajara Medina en su completísimo estudio ${ }^{15}$, existían profecías mesiánicas sobre la reconquista de Jerusalén -cuya pérdida había sido "provocada" por los pecados de los cristianos- por parte de un rey hispánico. Hasta Arnaldo de Vilanova, en general, las profecías habían asociado al conquistador cristiano con emperadores provenientes de Alemania y con la Casa de Francia; el médico catalán será el primero en derivarlo de la dinastía aragonesa, aunque "la difusión de esta idea en el profetismo peninsular aparece reflejada en muchos de los textos y autores de la tradición escatológica, desarrollándose con variantes no sólo en los visionarios aragoneses, sino también en los del reino de Castilla". ${ }^{16}$

Sabemos que entre 1305 y 1308 Arnaldo de Vilanova participó, junto con el rey Jaime II, de la organización de una cruzada. En 1309, y ante la presencia del Papa, lee sus "interpretaciones oníricas" en las que proyecta la imagen del emperador escatológico sobre la monarquía aragonesa-siciliana, concretamente sobre Jaime II y su hermano, Federico III $^{17}$, y anuncia los propósitos de vida cristiana y de conquista de Tierra Santa que tenían los reyes de Aragón y de Sicilia. Este mesianismo tendrá una inmediata consecuencia política: la expansión aragonesa hacia el sur peninsular pero también la proyección hacia Oriente, plasmada en la conquista por parte de Aragón de la ciudad santa de Jerusalén.

Profecías similares se desarrollarán ya en pleno siglo XIV con las obras de los franciscanos Juan de Rupescissa (m. 1365) y Francisco Eiximenis (1330-1409), quien en el capítulo CCXLVII ("De la dignitat del rey d'Aragó") de su libro Primer del Crestiá dice lo siguiente: "D'aquesta casa es profetat que deu aconseguir monarchia quasi sobre lo mon" y "speram per special profecia quen haven, segons que alcuns dien, que en breu passara en Affrica princep d'aquesta casa qui ha a humiliar e a destroir tota la secta d'Mahomet". ${ }^{18}$

Curiosamente, el libro de Mandevilla contiene varias alusiones a profecías acerca de la recuperación de Tierra Santa y demás regiones orientales:

\footnotetext{
"Et dizen algunos prophetas que vn princep seynnor d'occident ganará la tierra de promission con la ayuda de los christianos e fara cantar missa dejus este arbor de seth" (p. 56);

"Mas quoando plazdra a nuestro seynnor assi como aqueilla tierra a estado perdida por los peccados delos christianos assi sera eilla Reganada por la proeça d'eillos ala ayuda d'eill" (p. 60).

"Et assi dizen eillos [los moros] que eillos saben bien por prophecias que la ley de mahomet faldra assi como ha fecho la ley de los judios, que es fallida. Et que la ley del pueblo christiano durara ata la fin del mundo." (p. 78)
}

Mandevilla va más allá cuando pone en boca del sultán de Egipto una crítica a los cristianos que, pese a todo, deriva en un vaticinio a favor de una victoria contra los partidarios de Mahoma:

\footnotetext{
"(los cristianos) violan toda lur ley que jhesu christo lis auia dado e deuisada por lur saluament. Et assi por lures peccados han eillos perdido toda esta tierra que nos tenemos. Car nuestro dios la dio en nuestras manos no pas por nuestra fuerça Mas por lures pecados. Car nos sabemos bien por prophecias que christianos nos ganaran esta tierra quoando eillos seruiran lur dios mas deuotament" (p. 79).
} 
Curiosidad, casualidad o coincidencia es que las últimas tres citas no provengan, hasta el momento, de una fuente identificable, por lo que Christiane Deluz - estudiosa de las fuentes utilizadas por Mandevilla para la confección de su relato - las clasifica como composiciones originales o reflexiones personales del autor. Esto significa que dicha materia se halla presente sólo en el texto de Mandevilla y de allí el interés que pudo haber suscitado en la casa de Aragón.

Así quizás pueda interpretarse como un interés de tipo político el ejercido por el Libro de las Maravillas del Mundo sobre los miembros de la corona de Aragón cuando el infante Juan I solicitó las copias de los Viajes al rey de Francia y poco después, a su suegra, la duquesa de Bar. Que el tema tenía una gran actualidad en su reinado lo demuestra la inquietud manifestada por este monarca ante algunas afirmaciones de Eiximenis, que presentaban a la casa de Francia como como la única sobreviviente al final de los tiempos.

La profecía a la que alude se encuentra en el capítulo CCCCLXVI, Que han dit alguns dels regnes presents e de lur durada e de novell imperi, la última parte del Dotzè Llibre del Christià: "si tu me demanes aquestes presents senyories demunt dites de christians si durara fins a la fin del mon responte que no u se. es ver que alsguns passats han parlat d aquesta materia e han posat que apres la reformacio del mon la qual dien que $\mathrm{s}$ a fer dins lo centenar present en que comptan .mccclxxxv., apres $d$ aquest centenar so dicen se mudara la seu papal en Jherusalem, e dien que aquí haura novell papa e novell emperador, e seran abdos de linatge del jueus convertits a la fe ............. dien en cara que aço fet, tota potestat reyal cessara en lo mon, sino solament en la casa de França, qui durara longuament, por tal cant tost temps ha defesa la sancta Esgleya christiana e ha posat lo papa, nou veguades en la cadira papal, gitat d aquella per enamichs de la fe o per heretges o per tyrans o per antipapes ..." (Rubió y Lluch, 1908: 372). Por ello Juan I "le solicitó, por medio de Pedro de Artés, que se abstuviera de 'fer certes profecies, o que les hi demostri per art d'astrologia', lo cual puede dar cierta idea de cómo llegaron hasta la realeza los ecos de la inspiración apocalíptica."19.

Por último, y como una coincidencia más, recordemos que entre los libros solicitados por Juan I, se encontraba el Llibre de Merli, que si bien puede haber formado parte de sus lecturas sobre materia caballeresca, como el Lanzarote, es probable que haya interesado por sus componentes proféticos, en los que un leo ispanus realizaría la conversión de los infieles ${ }^{20}$.

Debido a la pérdida de los primeros folios del manuscrito aragonés, no es posible contar con la primera parte del texto. Dada la importancia de lo allí expuesto, transcribimos el pasaje del manuscrito francés más antiguo, considerado, a su vez, de la misma familia del que procedería la traducción aragonesa (Letts, 1953: 230). El citado pasaje - entre otros muchos extraídos de libros de viajes - sirve al historiador A. S. Atiya para marcar la función propagandística ejercida por los relatos de viajeros y peregrinos a Tierra Santa consistente en el llamado a la cruzada para la recuperación de los Santos Lugares:

\footnotetext{
"Bien doit estre delitable et fructueuse la terre qui fut arouzee du precieux sanc Ihesu Crist; cest la terre que nostre seigneur nous promist en heritage, et en la dicte terre vouloit il mourir comme saisi, pour la laissier en heritage a ses enfans. Par quoy chascun bon crestien qui pouoir en ha et de quoy, se deuroit pener et mectre en grant [trauail] de nostre surdit et droit heritage conquerir et mettre hors des mains aus mescreans et de lapropier a nous. Car nous sommes appelez crestiens de Crist, qui es nostre pere; et se nous sommes ses drois filz, nous deurons leritage que nostre pere nouz a laissie chalenger et oster des mains estranges. Mais au jour duy orgueil conuoitise et enuie ont ainsi les cuers des seigneurs terriens enflames et esprins, que il tendent plus a autrui desheriter que il ne font a chalenger et aquerre leur droit et propre heritage dessus dit. Et ceulz du commun, qui bonne volente en ont a mectre corps et auoir pour conquerir nostre susdit heritage, ne pueent riens faire senz les seigneurs souuerains. Car assemblee de communaute senz chief est comme tropel de brebis senz pasteur, qui sespandent et ne sceuent ou il doiuent aler, ne que ilz doiuent faire. Mais se il plaisoit a nostre saint pere le pape, car a Dieu plairoit il bien, que les princes terriens fussent de bon acort et auecques aucuns de leurs communs et voulsissent entreprendre le saint voiage doultre mer, ie cuide estre bien certain que en brief terme seroit la terre de promission reconciliee et mise es mains des drois hoirs, si comme de Ihesu Crist".
} 
Para finalizar, podría decirse que luego de contrastar algunos hechos históricos con pasajes de un libro cuya lectura es considerada actualmente por gran parte de la crítica especializada como de mero pasatiempo en cuanto exponente de curiosidades y rarezas, todo parecería indicar que no era esta la única lectura a la que había sido sometido en aquellas circunstancias. El Libro de las Maravillas del Mundo habría tenido en la corte de Aragón una lectura destinada a privilegiar cuestiones de carácter eminentemente informativo tendientes a confirmar noticias previas, condiciones de factibilidad y argumentos robustecedores en apoyo a intereses políticos con una larga tradición histórica. Una lectura similar, aunque un siglo más tardía, realizaría Cristóbal Colón en el marco de la expansión ultramarina llevada adelante durante el reinado de los Reyes Católicos. Curiosamente, Colón hará uso de los mismos libros (Marco Polo, Mandevilla) para argumentar favorablemente su proyecto considerado de dudosa realización.

$\mathrm{Al}$ respecto, su hijo Fernado asegura que

\footnotetext{
"llegado a decir las causas que movieron al Almirante a descubrir las Indias, digo que fueron tres: los fundamentos naturales, la autoridad de los escritores y los indicios de los navegantes. (...) Marco Polo, veneciano, y Juan de Mandavila, en sus Viajes, dicen que pasaron mucho más adentro del Oriente de lo que escriben Ptolomeo y Marino ..." 21 .
}

También son famosas las referencias de Andrés Bernáldez, en sus Memorias del Reinado de los Reyes Católicos ${ }^{22}$, al referirse al Almirante:

\begin{abstract}
"E sintió por qué vía se hallaría tierra de mucho oro, e sentió como este mundo e fírmamiento de tierra e agua es todo andable en derredor por tierra e por agua, segund cuenta Juan de Mandavilla: Quien toviese tales navíos e a quien Dios quisiese guardar por mar e por tierra, por cierto él podría ir e trasponer por el poniente, de en derecho al cabo de Sant Vicent, e bolver por Iherusalem e Roma, e dende a Sevilla, que sería cercar toda la redondez de tierra e agua del mundo" (p. 270),
\end{abstract}

o refiriéndose a la ciudad del Gran Khan:

\begin{abstract}
"E por la parte del austro, dexando la tierra de la Juana sobre la mano derecha, navegó pensando dar la buelta al derredor e correr, después de ver el cabo, la vía de su deseo, que era busca la provincia e cibdat del Cathayio, diziendo que la podría hallar por allí, que es en el señorío del Grand Can. De la cual se lee, segund dize Juan de Mandavilla, e otros que la vieron, que es la más rica provincia del mundo ..." (p. 307).
\end{abstract}

Tal vez debamos recordar la carta que Cristóbal Colón enviara a los Reyes Católicos en 1501, referida a las profecías tocantes a la "restituçión de la Casa Santa a la Santa Iglesia", o sea a la conquista de Jerusalén, la que finaliza: "El abad Johachin Calabrés diso que había de salir de España quien havía de redificar la casa del monte Sión" ${ }^{23}$. A propósito del tema, Alain Milhou (s/a: 207), afirma que las profecías mesiánicas protagonizadas por un rey de España que corrieron en la península ibérica desde fines del siglo XIII hasta la época de Colón, fueron divulgadas por franciscanos aficionados a la corte de Aragón. Ello lo lleva a relacionar el "mesianismo" con un clima de época y, en el caso particular de los Reyes Católicos, asociarlo con el tema de la propaganda monárquica.

En relación a este tema, Cris Zacher (s/a: 155-160) analiza la lectura que Colón hizo del texto de Mandevilla, la cual se resume en cuatro aspectos principales: 1) las aseveraciones respecto de la posibilidad de circunnavegar la tierra, 2) las descripciones de sociedades "salvajes" éticamente mejores que la europea, 3) el rescate de Tierra Santa y la reconstrucción del Templo en Jerusalén y 4) la narración en una primera persona retóricamente persuasiva que daba cuenta de los viajes como testigo ocular de los mismos.

Iniciamos este trabajo refiriéndonos al pedido que en el año 1380 hiciera el infante Juan [ de Aragón solicitando el libro de Juan de Mandevilla. Planteamos la hipótesis de los posibles intereses que el texto pudo haber deparado al joven monarca y a la casa de Aragón an general. Finalizamos el recorrido casi cien años después, hurgando en los escritos 
colombinos en los que coincidentemente perduran ideas e intereses similares en un contexto histórico y político delineado con mayor nitidez y en el que es posible contar con hechos que avalan nuestras lecturas. Como en un gran tapiz, la historia de la humanidad reconoce que puntadas dispersas pueden corresponder a una mișma hebra en el tejido, enseñándonos a entrever los vaivenes de ciertas ideas a través de los tiempos como indicio de su vitalidad.

Seminario de Edición y Crítica Textual (SECRIT-CONICET)

Universidad Nacional de La Plata

Argentina

\section{NOTAS}

1 Manuscrito M-III-7 de la Biblioteca de El Escorial.

2 Ediciones documentadas aunque no conservadas: 1483, 1500 y 1515 . Se conservan: Valencia, 1521 (dos ejemplares); Valencia, 1524 (un ejemplar); Valencia, 1531 (un ejemplar); Valencia, 1540 (un ejemplar) y Alcalá de Henares, 1547 (un ejemplar). Existe además un manuscrito francés del siglo XIV, conservado en la Biblioteca Nacional de Madrid con la signatura 9602.

3 C. Duret, Histoire admirable de plantes et herbes, Paris, 1605; C. Duret, Thrésor d'Histoire de langues de cest univers, Cologny, 1613. Ediciones modernas: F. Secret, "Une version oubliée des 'Voyages' de Jean de Mandeville, Studi Francesi, V, 1961, pp. 480-483 y A. Rossebastiano Bart: "La 'langue romanesque' del 'Voyage d'outremer' di Jean de Mandeville, Aevum, LVIII, 1984, pp. 287-300.

4 Todas las citas pertenecen a esta edición, y las negritas son mías.

5 Se trata, en realidad, de dos obras: El purgatorio de San Patricio y el Libro de Mandevilla.

6 Editada por John Nitti, Juan Fernández de Heredia's version of the "Libro de Marco Polo", Madison, Hispanic Seminary of Medieval Studies, 1980.

7 John Nitti y Lloyd Kasten, "Flor de las ystorias de Orient: Escorial Z.I.2" en The electronic texts and concordances of Medieval Navarro-Aragonese Manuscripts, Madison, Hispanic Seminary of Medieval Studies, 1997, folio 1r. Este texto, al igual que el libro de Marco Polo fueron copiados por Fernando de Medina entre 1385 y 1393.

8 Véase Dawn E. Prince (ed.), The Aragonese Version of Brunetto Latini's Libro del trasoro, Madison, Hispanic Seminary of Medieval Studies, 1995 , p. xi.

9 La fecha del documento es 15/10/1379. Las cursivas son mías.

10 Para el tema de las traducciones científicas al catalán en el siglo XIV, véase Julio Samsó: "Traducciones científicas arabo-romances en la península ibérica", Actes del VII Congrés de l'Associació Hispànica de Literatura Medieval (Castelló de la Plana, 22-26 de setembre de 1997), Santiago Fortuño Llorenes y Tomás Martínez Romero (eds.), Vol. I, pp. 199-231.

$11 \mathrm{Al}$ respecto, véase Julio Rey Pastor y Ernesto García Camarero, La cartografía mallorquina. Madrid, Departamento de Historia y Filosofía de la Ciencia, Instituto Luis Vives, Consejo Superior de Investigaciones Científicas, 1960. El estudio contiene una catalogación minuciosa de mapas de los que se tiene noticia, así como también una descripción de los conservados en distintas bibliotecas de Europa y América, lo cual permite adquirir una cabal idea de la importancia que a nivel científico significó el desarrollo de la cartografía mallorquina.

12 El cambio de forma introducido por los mapas portulanos, de circular a rectangular, indicaría que como astrónomo, su autor muy probablemente aceptara la esfericidad de la tierra.

13 Véanse Amada López de Meneses: "Los consulados catalanes de Alejandría y Damasco en el Reinado de Pedro el Ceremonioso", Estudios de Edad Media de la Corona de Aragón, vol. VI, Zaragoza, CSIC, Escuela de Estudios Medievales, 1956, pp. 83-183; "Pedro el Ceremonioso y Palestina", en Homenaje a Don José María Lacarra de Miguel en su jubilación del Profesorado. Estudios Medievales III, Zaragoza, Anubar Ediciones, 1977, pp. 269-290 y Vicente Salavert y Roca: "La expansión catalano-aragonesa por el Mediterráneo en el siglo XIV" en La investigación de la Historia Hispánica del siglo XIV. Problemas y Cuestiones. Actas del I Simposio de Historia Medieval, 20-23 de marzo de 1969, Madrid-Barcelona, CSIC, 1973, pp. 17-37.

$14 \mathrm{Al}$ respecto, véase el capítulo de Javier Fernández Conde "La Santa Sede y la Cruzada Española", en Ricardo García Villoslada (director): Historia de la Iglesia en España. Tomo II- $2^{\circ}$. La Iglesia en la España de los siglos VIII-XIV, Madrid, Biblioteca de Autores Cristianos, 1982, pp. 361-371.

15 José Guadalajara Medina, Las profecías del Anticristo en la Edad Media. Madrid, Gredos, 1996. Véase, en particular, el cap. IV, 1: Profetas y vaticinios escatológicos en el Reino de Aragón.

16 Guadalajara Medina, op. cit. pp. 205-206. Véase además el capítulo 'Mesianismo y especulación profética en Arnaldo de Vilanova' (pp. 195-206).

17 Se trata de su escrito Interpretatio facta per magistrum Arnaldum de Vilanova de visionibus in somnis 
dominourun Iacobi Secundi, regis Aragonum, et Friderici Tertii, Regis Siciliae, eius fratis. Véase también Menéndez y Pelayo, Obras Completas. Historia de los Heterodoxos Españoles, Tomo III, Buenos Aires, Espasa Calpe Argentina, S.A., 1951, pp. 163-204.

18 Guadalajara Medina señala que en el capítulo CCLVI Eiximenis deja constancia del año en el que está escribiendo (1379). Un lector anónimo ha consignado al margen la fecha de 1492, posible fecha en que manejaba la obra, y ha subrayado y marcado en especial fragmentos del capítulo CCXLVII. Este hecho estaría demostrando la importancia de estas profecías y la vigencia hasta la época de los Reyes Católicos.

19 Guadalajara Medina, op. cit., p. 213; Rubió y Lluch, 1908, doc. CCCCXVIII del 12/12/1391.

20 El Llibre de Merli es solicitado por Juan I el 24 de noviembre de 1391 (doc. CCCXXXVIII, en Documents ..., 1921). Para las profecías de Merlín en Aragón véase Guadalajara Medina, op. cit., pp. 225-226.

21 Fernando Colón, Historia del Almirante de las Indias Cristobal Colon. México, Editorial Latino Americano, 1958, pp. 32 y 35. Citado por Pilar Liria Montañés en su edición del libro de Mandevilla, p. 19.

22 Andrés Bernáldez (Cura de los Palacios), Memorias del Reinado de los Reyes Católicos. Manuel Gómez Moreno y Juan de M. Mata Carriazo (eds.), Madrid, Real Academia de la Historia, 1962, pp. 234, 269-270, $307-308,315$ y $318-319$.

23 Colón, Cristóbal, Textos y documentos completos. Consuelo Varela (ed.), Madrid, Alianza Editorial, 1989, p. 207.

\section{BIBLIOGRAFÍA}

Actas del Primer Encuentro Internacional Colombino (noviembre de 1988), Consuelo Varela (ed.). Turner, s/a. Atiya, Aziz Suryal, The Crusade in the Later Middle Ages. London, Methuen \& Co. Ltd., 1938.

Bernáldez, Andrés (Cura de los Palacios), Mernorias del Reinado de los Reyes Católicos. Manuel Gómez Moreno y Juan de M. Mata Carriazo (eds.). Madrid, Real Academia de la Historia, 1962.

Cacho Blecua, Juan Manuel, El Gran Maestre Juan Fernández de Heredia. Zaragoza, Caja de Ahorros de la Inmaculada de Aragón, 1997.

Colón, Cristóbal, Textos y documentos completos. Consuelo Varela (ed.), Madrid, Alianza Editorial, 1989.

Colón, Fernando, Historia del Almirante de las Indias Cristobal Colon. México, Editorial Latino Americano, 1958.

Cortés Arrese, Miguel, "Algunos aspectos del mecenazgo artístico de Juan Fernández de Heredia" en Juan Fernández de Heredia y su época. IV Curso sobre Lengua y Literatura en Aragón, Aurora Egido y José María Enguita (eds.). Zaragoza, Institución Fernando el Católico, 1996, pp. 31-56.

Deluz, Christiane, Le Livre de Jehan de Mandeville, une "géographie" au XIVe. s. Publications de l'Institut d'études médiévales, Textes, Études, Congrès, n8, Louvain-la-Neuve, 1988.

Duret, Claude, Histoire admirable de plantes et herbes. Paris, 1605.

- Thrésor d'Histoire de langues de cest univers. Cologny, 1613.

Eco, Umberto, Arte y belleza en la estética medieval. Barcelona, Lumen, 1997.

Entwistle, William J., "The Spanish Mandevilles". Modern Language Review, XVII, 1922. pp. 251-257.

Fick, Bárbara, El libro de viajes en la España medieval. Santiago de Chile, Seminario de Filología Hispánica, Editorial Universitaria, 1976.

García Villoslada, Ricardo (director), Historia de la Iglesia en España. Tomo II-2. La Iglesia en la España de los siglos VIII-XIV, Madrid, Biblioteca de Autores Cristianos, 1982.

Gil, Juan (ed.), El libro de Marco Polo anotado por Cristóbal Colón. Madrid, Alianza Universidad, 1987.

Gómez Moreno, Manuel y Mata Carriazo, Juan M. de (eds.), Bernáldez, Andrés. Memoria del Reinado de los Reyes Católicos. Madrid, Real Academia de la Historia, 1962.

Guadalajara Medina, José, Las profecías del Anticristo en la Edad Media. Madrid, Gredos, 1996.

Hauf i Valls, Albert Guillem, "Texto y contexto de la Flor de las historias de Oriente: un programa de colaboración cristiano-mongólica", en Juan Fernández de Heredia y su época. IV Curso sobre Lengua y Literatura en Aragón, Aurora Egido y José María Enguita (eds.). Zaragoza, Institución Fernando el Católico, 1996.

Ledesma Rubio, María Luisa, "Juan Fernández de Heredia en el marco de la orden del Hospital de San Juan de Jerusalén”, en Juan Fernández de Heredia y su época. IV Curso sobre Lengua y Literatura en Aragón, Aurora Egido y José María Enguita (eds.). Zaragoza, Institución Fernando el Católico, 1996, pp. 17-30.

Letts, Malcolm (ed.), Mandeville's Travels. Texts and Translations. T. II, London, The Hakluyt Society, 1953.

Liria Montañés, Pilar (ed.). Libro de las maravillas del mundo, de Juan de Mandevilla. Zaragoza, Caja de Ahorros de Zaragoza, Aragón y Rioja, 1979.

López de Meneses, Amada, "Los consulados catalanes de Alejandría y Damasco en el Reinado de Pedro el Ceremonioso", Estudios de Edad Media de la Corona de Aragón, vol. VI, Zaragoza, CSIC, Escuela de Estudios Medievales, 1956, pp. 83-183.

- "Pedro el Ceremonioso y Palestina", en Homenaje a Don José María Lacarra de Miguel en su jubilación del Profesorado. Estudios Medievales III, Zaragoza, Anubar Ediciones, 1977, pp. 269-290. 
Menéndez y Pelayo, Marcelino: Obras Completas. Historia de los Heterodoxos Españoles, Tomo III. Buenos Aires, Espasa Calpe Argentina, S.A., 1951.

Milhou, Alain: "II Sesión. Mesa Redonda", en Actas del Primer Encuentro Internacional Colombino (noviembre de 1988), Consuelo Varela (ed.). Turner, s/a.

Millás Vallicrosa, José María, 1960. Nuevos Estudios sobre Historia de la Ciencia Española, Barcelona, CSIC, Instituto Luis Vives de Filosofía.

Nitti, John y Kasten, Lloyd, "Flor de las ystorias de Orient: Escorial Z.I.2" en The electronic texts and concordances of Medieval Navarro-Aragonese Manuscripts, Madison, Hispanic Seminary of Medieval Studies, 1997.

Nitti, John, Juan Fernández de Heredia's version of the "Libro de Marco Polo", Madison, Hispanic Seminary of Medieval Studies, 1980.

Patterson, Lee, Negotiating the Past. The Historical Understanding of Medieval Literature. Madison, The University of Wisconsin Press, 1987.

Prince, Dawn E. (ed.), The Aragonese Version of Brunetto Latini's Libro del trasoro, Madison, Hispanic Seminary of Medieval Studies, 1995.

Rey Pastor, Julio y García Camarero, Ernesto, La cartografía mallorquina. Madrid, Departamento de Historia y Filosofía de la Ciencia, Instituto Luis Vives, Consejo Superior de Investigaciones Científicas, 1960.

Richard, Jean, Les récits de voyages et de pèlerinages. Turnhout-Belgium, Typologie des Sources du Moyen Age Occidental, Brepols, 1985.

Rossebastiano Bart, Alda, 'La 'langue romanesque' del 'Voyage d'outremer' di Jean de Mandeville, Aevum, LVIII, 1984, pp. 287-300.

Rossebastiano, Alda, La tradizione ibero-romanza del "Libro de las maravillas del mundo", di Juan de Mandavila. Alessandria, Studi, Biblioteca Mediterranea, Edizioni dell'Orso, 1997.

Rubió y Lluch, Antoni, Diplomatari de l'Orient Català (1301-1409). Barcelona, Institut d'Estudis Catalans, 1947.

- Documents per l'Historia de la Cultura Catalana Mig-eval, vols. I y II. Barcelona, Institut d'Estudis Catalans, Palau de la Diputació, 1908-1921.

Salavert y Roca, Vicente: "La expansión catalano-aragonesa por el Mediterráneo en el siglo XIV" en La investigación de la Historia Hispánica del siglo XIV. Problemas y Cuestiones. Actas del I Simposio de Historia Medieval, 20-23 de marzo de 1969, Madrid-Barcelona, CSIC, 1973, pp. 17-37.

Samsó, Julio: "Traducciones científicas arabo-romances en la península ibérica", Actes del VII Congrés de l'Associació Hispànica de Literatura Medieval (Castelló de la Plana, 22-26 de setembre de 1997), Santiago Fortuño Llorenes y Tomás Martínez Romero (eds.), Vol. I, pp. 199-231.

Secret, F., "Une version oubliée des 'Voyages' de Jean de Mandeville, Studi Francesi, V, 1961, pp. 480-483.

Taylor, Barry, "Los libros de viajes en la Edad Media Hispánica: Bibliografía y Recepción", en Actas do IV Congresso da Associação Hispánica de Literatura Medieval, I. Lisboa, Cosmos, 1991.

Waters Bennett, Josephine, The rediscovery of Sir John Mandeville. New York, Modern Language Association, 1954.

Zacher, Cris: "How Columbus read Mandeville's travels", Actas del Primer Encuentro Internacional Colombino (noviembre de 1988), Consuelo Varela (ed.), Turner, s/a., pp. 155-160.

Zumthor, Paul, Essai de poétique médiévale. Paris, Editions du Seuil, 1972. 